Dr. Manuel Núñez Regueiro (*)

\title{
Cartas a don Enrique Molina
}

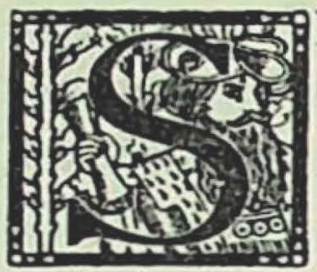

EÑOR profesor Dr. Enrique Molina, Universidad de Concepción, Chile. Mi dilecto amigo: Recibí su Confesión filosófica y Llamado de superación a la América hispana. Conocía la primera, no así el "llamado" a la ansiada superación. Pero lo suponía tratándose de un espíritu que sabe volar tan alto en el cielo del pensamiento americano. Comparto decididaniente la justeza de los juicios que han merecido su definición personal en el campo rico y nunca terminado de sembrar de la verdadera filosofía viviente. La claridad diáfana de su bello discurso, su fluidez de estilo vestido de sencilla elegancia, la hondura del análisis, la sugestión de las ideas, dan a su obra una atracción que merece destacarse en el Olimpo de nuestros mejorés guías y pensadores. Por todo ello cordialmente lo felicito. Su obra tiene sello de trascendente inmortalidad, porque vive en el medio adecuado a las cosas del espíritu, cuyos valores son nermanentes. Huye de lo efímero y va a buscar la verdad en el fondo de la vida, reflexionando serenamente, con sensibilidad y simpatía. Estamos en momentos en que es preciso definirnos; una definición es la mejor imagen del hombre tal lo contemplo a Ud. Bellamente encaramado en las cumbres alumbradas por el sol del Verbo Eterno. Quien de ese modo trabaja, debe sobrevivir a las mudanzas del tiempo, a los

(*) El Dr. Manuel Núñez Reguciro, autor de la carta que transcribimos, es un distinguido publicista y filósofo, director del Círculo de Altos Estudios de Rosario (República Argentina) y profesor de la Universidad Nacional del Litoral que funciona en esa misma ciudad. 
efímeros azares de la batalla del hombre contra el hombre. Lo importante es no pleitear con Dios en estos momentos de trágica transgresión de la inteligencia, desorbitada y vacía de savia ontológica. Lo acompaño sinceramente en esa noble batalla del Príncipe de la luz contra Belcebú, rey de las tinieblas. Debemos, como una antorcha, consumirnos alumbrando. Tal es nuestro más alto y saludable destino, si queremos comparecer ante el Juez justo e inapelable del poder trascendente que gobierna el Universo, gozosos de haber cumplido con nuestro deber. No hay otro sendero a toda posible superación del espíritu. América debe gritar bien alto la belleza de su actitud, de cómo sabe conducirse ejemplarmente en esta hora crucial de nuestra civilización. Los buenos conductores se llaman Roosevelt, etc. No hagamos cuestión de nombres. También es tarea de auténticos fi'ó. sofos y pensadores guiar a los pueblos; por todo ello alabo su obra y me complazco en felicitarlo cordialmente, con la devoción sincera de su amigo y admirador.-Manuel Núñez Regueiro.

Rosario, Santa Fe, enero, 1943.

\section{CARTA DEL DR. CORIOLANO ALBERINI (*)}

Buenos Aires, mayo 31 de 1941.

Señor Dr. don Enrique Molina.

Mi muy estimado amigo y colega:

Acabo de leer su libro sobre Lo espiritual en la vida humana. No se imagina con cuanto interés lo he estudiado. Se trata, sin duda, de la mejor de sus obras, entre otras no menos buenas. Ya sabe que

(*) El doctor Coriolano Alberini es un conocido y prestigioso profesor de Filosofía de las Universidades de Buenos Aires y de La Plata; ha sido durante muchos períodos Decano de la Facultad de Filosofía y Letras de la primera y su Vicerrector. 
siempre lo he considerado a Ud., junto con Carlos Vaz Ferreira, el filósofo más personal entre los hispanoamericanos. Recordará Ud. nuestras conversaciones. En ellas muchas veces se ha aludido al filosofismo a base de erudición unilateral. Casi toda la publicación filosófica latinoamericana es mera glosa, refrito académico y gaceta. La erudición en filosofía no es sino un medio. Lo importante es lo otro, precisamente lo que yo encuentro en Ud. y en el colega uruguayo. Si fuera más afecto a la pluma, diría otras cosas.

Conservamos aquí un recuerdo gratísimo de su reciente visita. Por lo que a mí toca, espero que pronto tendré el placer de verle allí, en su Universidad de Concepción, que Ud. ha creado.

Reciba un saludo cordialísimo de su amigo y admirador.Coriolano Alberini.

\section{CARTA DEL DR. ANTONIO SAGARNA}

Buenos Aires, mayo 10 de 1938.

Señor Dr. Enrique Molina, Concepción (Chile).

Muy estimado Rector y amigo:

Anoche concluí la lectura del meduloso y bello libro $D e$ lo espiritual en la vida humana, que Ud. ha tenido la fina atención de obsequiarme y que le agradezco vivamente.

Las meditaciones de Ud., caro don Enrique, me dejan, como ciertos ensayos de Duhamel y Maeterlink ( La posesións du Monde y La Sagesse et la Destinée, p. e.), una sensación de altura, de serenidad y de optimismo, pues, por encima y más allá de la dialéctica, del análisis de doctrinas, de los alegatos más o menos fundados en pro o en contra de tal o cual tesis filosófica, se respira en ellos y en Ud. un como rezumo de vidas plenas, armoniosas que buscan su verdad sin prejuicios, sin fatiga y sin intolerancias; que en cada dato "de aquí abajo" que sale al paso de sus investigaciones y meditaciones, ven el eslabón de otros datos que pueden aparecer o no, pero que no ocultan, ni empecen ni anublan la posibilidad y la espe- 
ranza de una causa de causas que, aún como ilusión, es un dulce consuelo y aliento en el peregrinar.

Don Francisco de Veyga, uno de nuestros más ilustrados hombres de ciencia: biólogo, médico, psicólogo, psiquiatra, criminólogo, sociólogo, etc., dice en su libro La Inteligencia y la Vida:

"Las manifestaciones $\mathrm{u}$ operaciones de orden orgánico podrán ser todas íntegramente, de carácter material o energético - no liscutamos mayormente sobre el particular-, pero eso no implica que sean resultado de condiciones fortuitas fijadas por la materia, el simple dispositivo accidental de los elementos que entran en juego, o la entrada en acción de tales o cuales propiedades de cuerpos accidentalmente colocados allí, la simple coincidencia de actuación".

"Ahora bien, estos mecanismos no andan solos. No desfigurernos los hechos, no nos engañemos a nosotros mismos, buscando ser fieles al engaño de otros. Aquí hay algo más que un juego de elementos: Hay un orden de cosas, un dispositivo, situaciones muy especiales según las cuales los tales resortes y los tales trabaios están llamados a llenar una necesidad determinada. ¿Quién ha establecido esa armonía entre la función desempeñada y la necesidad sentida? ¿Quién la satisfacción de ser llenada? No es cuestión de inventar o suponer por espíritu de sistema o de simple presunción. Hay elementos retroactivos en acción y miras teológicas por delante".

Hago la cita, quizá un poco extensa, porque veo en 'su magnífico estudio una reafirmación de ese criterio disconforme con el mero determinisnıo positivista, y así -entre otras expresiones- es categórica ésta de la página 159.

“Aunque no encontremos jamás el espíritu sin que esté adscripto a un cuerpo, lo propio de su naturaleza es un quid que no se explica totalmente por sus antecedentes materiales, como se ve en el milagro de brotar ideas nuevas", y en realidad todo el final hermoso del libro y todo el libro, en una palabra, señero como el espíritu del autor.

Sus reflexiones sobre el carácter y, particularmente sobre el carácter americano, merecen una reedición muy amplia en toda esta tierra del ensueño bolivariano, del idealismo huero; del nacionalis- 
mo agresivo y de dolorosa incapacidad para asumir la efectiva soberanía. En un estudio sobre Alberdi - que Pepe Ingenieros publicó-; en una conferencia limeña sobre "Paralelismo de la Evolución Económica y Espiritual Argentina"; en una conferencia en Luján sobre el "Centenario de Ayacucho" y en varias otras oportunidades expresé modos de ver coincidentes con los de Ud.

El carácter, según la fórmula de Fouillée, magníficamente integrada por Ud., con los recaudos del "valor", "el sentido de responsabilidad", el "dominio de sí mismo", la "capacidad de atención" y la "claridad de pensamiento", no abundan en Perú, Chile, Argentina, Uruguay y Paraguay, que es donde conozco directamente algo; el carácter se confunde frecuentemente con la testarudez, la falsa austeridad, la reacción pronta y agresiva; y la espiritualidad, a los que llaman también idealidad o idealismo, asume la segunda de las formas que Ud. menciona (capítulo VIII); el "positivismo", el "materialismo", el "sensualismo" de "esta hora prosaica" son denunciados por muchos que no han aportado ni a su país, ni a su familia, ni a sí mismo otra cosa que gimoteos y charlas de bohemios falsificados. Estudio metódico, disciplina, perseverancia son factores que no cuentan en la formación de una gran parte de la juventud iberoamericana que no se la ve en los conciertos, en las exposiciones, en las conferencias, ni lee revistas ni libros de verdadero contenido espiritual.

Tengo la esperanza de prontas y eficaces reacciones; del exceso del mal saldrá el remedio.

Una vez más, muchas gracias por su lindo regalo y reciba un afectuoso apretón de manos de su amigo.-Antonio Sagarna.

\section{DOS CARTAS DE DON ERNESTO BOERO LILLO}

Santiago, 21 de diciembre de 1943.

Don Enrique: Para llegar hasta usted con el modestísimo presente de mis palabras y mis recuerdos, deliberadamente he estado esperando que cesaran los inñúmeros homenajes que le han rendido 
a usted por sus diez lustros de educador sin par, homenajes grandio. sos por sus proporciones algunos, emocionantes por la belleza de sentimientos otros, y todos, de una hondura de significado no fácil de volver a ver en la vida educacional y social chilena.

Puede haber en esta actitud mía, o un fondo de timidez, de retraimiento, o - ¿paradoja del subconsciente? - un deseo de llegar solo, sin formar parte de ningún coro, por magnífico que fuera.

Pues bien, porque ya me lo imagino en el refugio de su hogar y de su Universidad, serenado su espíritu puesto a prueba de tantas emociones, llego yo para decirle que con usted he estado y vivido todos esos instantes supremos de su vida de maestro y he sentido henchírseme el alma de satisfacción y alegría al ver que a su alrededor por fin se manifestaba en forma brillante y unánime la gratitud humana. ¡Cuánta verdad hay en la frase de Van Dike: “el maestro tiene el más mísero de los salarios y la más espléndida de las recompensas morales!"

Vivos están en mis recuerdos aquellos tiempos cuando usted llegó a hacerse cargo de la rectoría del Liceo de Concepción. Formaba parte yo de un curso no muy brillante en sus estudios oficiales. pero con una acentuada inquietud espiritual. Con usted llegó un nuevo e intenso soplo de vida a la ciudad entera. Su acción no se circunscribió al aula liceana. Para usted, maestro y ciudadano - iresurrección de la Grecia inmortal!-, el aula fue la ciudad toda e hizo remover tantos espíritus de valer que dormían bajo la inquietud de sus afanes cotidianos. Así, gracias a su impulso nació el Ateneo de Concepción, y, luego después la Extensión Secundaria y Universitaria. El Teatro Concepción fue entonces escenario de reuniones públicas de la más alta calidad artística. De un salto, al conjuro mágico de su palabra persuasiva, Concepción dejó de ser un pueblo huraño, con positivos pero avaros valores espirituales, y su juventud rió, co. mo nunca antes lo había hecho, y con ella toda la ciudad vibró en las más bellas alegrías de las fiestas primaverales.

Los muchachos del liceo de aquellos años - recuerdo a Francisco Santibáñez, a Rolando Merino, Francisco Aguilera-, bajo los co- 
rredores conventuales, pobremente conventuales, del liceo viejo, comentábamos, con frases admirativas, tan sorprendente eclosión - perdone este galicismo, don Enrique- del espíritu y del intelecto penquista. Brillaron nombres como los de Ignacio Verdugo Cavada, Exequiel de la Barra, Luis David Cruz Ocampo, Abraham Valenzuela (padre e hijo), Enrique Marshall, Carlos Quilodrán Roa, Juan Eduardo Moreno, y varios más. $\mathrm{Y}$ junto a éstos la palabra sabia, con un poder de atracción único, de "nuestro Rector", de "Don Enrique", que era oro puro, de la más alta ley, para nuestras ansias adolescentes. ¡Con qué recogimiente lo escuchábamos, don Enrique! Los días aciagos de la revolución francesa, los "vivíamos" bajo la magia de su disertación, y salíamos de clase con la emoción silenciosa que produce lo grandemente trágico, y lo enormemente heroico, lo magníficamente sublime. Y usted - ya estábamos en el edificio nuevo del liceoen los corredores del tercer piso, desde donde se domina la ciudad, terminada la clase, continuaba con nosotros, en un grupo familiar por la cordialidad, como de padre e hijos, hablándonos de la grandeza del alma humana, de lo inconmensurable del espíritu del hombre, de la fe creadora e impulsadora de grandes acciones, del idealismo, de la dignidad, del más allá, de Dios... Y así, hasta que la llegada del crepúsculo nos hacía disolver el grupo atento que formábamos y regresábamos a casa, meditabundos... con pocas ganas de estudiar y resolver los guarismos matemáticos de don Pedro Muñoz, o, en vez de tomar la Física Experimental de Ziegler y Gostling, para la rendición de cuentas que al día siguiente nos exigiría don Humberto Vergara, Rodó con su Ariel y Plutarco con sus Vidas paralelas, nos absorbía el espíritu, el alma y la voluntad

"Recordar el pasado es como pasar bajo el arco de la mueite", dice en uno de sus libros el marqués de Bradomín. Este ayer que hoy recuerdo, para mí es como empezar de nuevo a vivir.

$Y$ no quiero recordar más, don Enrique, porque sería cuento de nunca acabar.

He querido solamente, con estos recuerdos, hacerle ver a usted lo que usted era para nosotros. $\mathrm{Y}$ hoy, después de tantos años - ¡no 
quiero saber cuántos!-, usted es todo y eso y mucho pero mucho más... Y aquí la palabra se hace pobre y torpe, pequeña, incapaz, y prefiere callar.

Con todo lo dicho y lo no dicho, va mi espíritu toral, rendidamente hacia usted.-Erncsto Boero Lillo.

\section{* * * *}

Santiago, 29 de diciembre de 1953.

Señor don Enrique Molina, Concepción.

Don Enrique: Al informarme por la prensa que la Ilustre $\mathrm{Mu}-$ nicipalidad de Concepción había instituido, en forma permanentemente anual, un Premio de Arte y Literatura, y que, al discernirlo por primera vez, hace algunos meses, había recaído esta recompensa en usted, fueron para mí momentos de dubitación de si hacerle presente mis congratulaciones... ¿Y por qué esta indecisión cuando yo, desde mis años de liceo - jtan presentes los tengo!-, he seguido punto por punto, casi apasionadamente, la trayectoria de su vida? El hecho fue que pasaron días y más días sin que mi palabra, débil interpretadora de mis sentimientos, llegara a concretarse en la escritura. ¿Qué ocurrió en mí? pregúntome esta tarde al leer en "Atenea" un comentario acerca de este laurel que la ciudad de Concepción ha puesto en sus sienes de escritor. Sin duda que ha mediado un fenómeno anímico proveniente quizá de qué imperativo del subconsciente, que ahora, a medida que le escribo, trato de encontrar y analizar. Para ello, me dejo llevar por la corriente de recuerdos y reflexiones.

La ciudad de Concepción - la verdad es que tendría que decir al país- débele a usted mucho (restringido se me hace el concepto cuantitativo de esta palabra). Con la vara luminosa de su espíritu usted despertó y encauzó las voluntades de la ciudad, luego de haber arribado a sus playas hace treinta y nueve años. $Y$ desde entonces, sin desmayos, y en continuada lucha con los precarios elementos ma- 
teriales, con la vacilante fe de los humanos y con los acontecimien tos imprevisibles de lo extrahumano, fue surgiendo la Universidad, y a influjo de ella, al correr del tiempo, fue creándose en ia ciudad una fisonomía universitaria, en su espíritu y en su carácter. Si se sintetizara en gráficos el nacimiento, niñez, adolescencia y juveñtud de la Universidad penquista, a manera de cinta cinematográfica, sería como seguir el desarrollo de un cuento de maravillas, no de mitológicas hadas como protagonistas, sino que de mortales seres de carne y hueso. Es tema para un libro emulador, de esos que se po:en en manos juveniles como inspiradores de ejemplos que seguir y metas que aspirar.

¡Y tanto y tanto más!..

Pero, ¿a qué viente esto, tan sabido, pero nunca lo suficientemente repetido? ¿A qué viene esto, al parecer sin ilación ni atingencia con el caso tan personal de mi retardo en manifestar a usted mi parecer acerca del Premio de Literatura?

No obstante... vayan otras consideraciones más, pues, don Enrique, como le he dicho al comienzo, trato en estos mismos momentos en que le escribo de encontrar y de analizar los motivos que me hicieron caer en omisión suspendiendo mi primer intento de voluntad.

Era yo alumno del liceo cuando circuló de boca en boca de la muchachada pobladora de los claustros escolares que, por fallecimiento de don Pedro N. Cruz Silva, llegaría a tomar las riendas de nuestra vieja y querida casa de estudios el que era Rector del Liceo de Talca. Aunque adolescentes, empinándonos por saltar la valla de cuarto a quinto años, no vivíamos ajenos al movimiento y realidad intelectual del país - iy del mundo!-, y, cierto que con poca precisión, estábamos informados que nuestro nuevo Rector había efectuado una gran labor en aquel plantel educacional talquino. Además sabíamos de unas conferencias suyas dadas en la Universidad capitalina, en defensa del liceo, del sentido humanístico de su enseñanza, duramente atacado por el que iba a ser más tarde el autor de la Historia de Chile, don Francisco Antonio Encina. Estos antecedentes 
nos llenaban de satisfacción, pues venía hacia nosotros no un profesor cualquiera, sino un hombre de principios arraigados, de sólida cultura, y, por sobre todas las virtudes, un luchador. O sea, un espíritu de estudio y de acción a la vez, cualidades muy raro de ver juntas, y que es lo que la juventud más aplaude y más admira.

Mientras esperábamos con la muy humana curiosidad de conocer la figura física del que ya era oficialmente nuestro Rector - su voz, su andar, sus ademanes, su genio, su uso o no uso de las palmetas disciplinarias-... nos fuimos en busca de sus libros. La biblioteca liceana, ubicada en aquellos tiempos en una pequeña sala, ahita de volúmenes, contigua al zaguán de entrada por calle Caupolicán -viejos muros quizás ya demolidos-, nos proporcionó Filosofía americana, bello volumen de tapas rojas, en pulcra edición francesa de Garnier. En las flacas librerías de la época, encontramos Educación contemporánea. Supimos con ellos, adentrándonos algo a ciegas, del pragmatismo de William James, y del "idealismo práctico", que fue para nosotros como un ventanuco abierto hacia un luminoso campo de vocaciones perfiladas entre brumas. Principios y fines de la existencia humana, como individuo y como colectividad, nos hicieron pensar que la vida era más vasta y más profunda que las horas afectivas del hogar y que la sabiduría elemental del aula.

Doy un salto en el tiempo, el comprendido entre 1915 al 16, que, aunque es el lapso más significativo y trascendente, pues de ahí parte el camino ascensional de la cultura educacional en Concepción, no tiene atingencia con el desarrollo de mi pensamiento y de los hechos que lo informan en esta especie de exposición reflexiva que hago para encontrarme a mí mismo en un insignificante - para los demás, tal vez, para mí no- acto volitivo que origina esta carta.

Pues bien, algún tiempo después, lejos del paternal alero liceano, y lejos de los lares nativos, viviendo en otro clima, de distinto sol y distintas gentes, cada cual de aquel grupo de muchachos, con los materiales de su propia personalidad, algo inestables aún, levantó tiendas unipersonales que lo guarecieran de las ráfagas de vientos 
encontrados que vienen de fuera y también de dentro de uno mismo. Y quedó dispersa la hermandad nacida y fortalecida a la sombra del verde ondulante de los pinares del Caracol.

Ya en esta parte de "mi historia" no me queda más que hablar en forma individual.

Desde acá, y a través del tiempo, he seguido, como le he dicho, casi apasionadamente, la trayectoria de su vida. $\mathrm{Y}$ puedo decir que desde aquellos años en que usted, en una sala del tercer piso del liceo, nos hablaba de los grandes hechos de la humanidad, no ha habido en mí ningún intervalo de tiempo sin que dejara de contar con la presencia y la palabra del maestro. Ni cuando dije adiós a la casa del liceo, ni cuảndo alisté mis bártulos estudiantiles, ni cuando mis ojos vieron cómo los campos nativos se esfumaban a la distancia, así como ahora, en estos momentos, frente a mi fiel Underwood, con la vista y el corazón algo cansados... ¿Dónde está el origen de este influjo suyo? ¿Dónde, de su permanencia en las generaciones que lo conocieron a través del espacio y del tiempo? Doctos biógrafos lo dirán, y ya lo han dicho algunos. Yo, en cambio, más objetivamente y más simplemente, me detengo sólo en los hechos adheridos a mí. La línea o parábola ininterrumpida, tuvo su punto de iniciación hace treinta y nueve años, tras los muros grises del liceo, desde la palabra y actitud del maestro, que siguió por las arterias de la ciudad, continuó en el jardín universitario hecho de piedras y rosas, prosiguió en la tribuna académica, y, perforando distancias, se irradió en sus quince o veinte libros, que aquí los tengo, dóciles a mis requerimientos. El libro es una cátedra en perennidad. La palabra, la voz interna, el pensamiento, están en él fijos y móviles a la vez. Es fuerza aparentemente quieta, mas, tremendamente dinámica. Estable en su sonido y en su esencia, es vigorosa en su expresión transmisora de energías. Paradójico es el milagro del libro. Y su obra literaria, don Enrique, es lo que ha contribuido a tenerlo presente, junto a los recuerdos de nostálgico sabor, añoranzas que dan a esta cátedra sin muros que la constriñan, una dulzura de melancolía. 
Sus libros, don Enrique -obra densa y constructiva-, han ido creando un fuerte "debe" en los organismos rectores de la república. El Estado, con el galardón que ha instituido para sus mejores escritores, está en mora con usted. La creación y formación de caracteres en médula y sangre, vale más, mucho más, que la creación literaria de entes de ficción. Así, lo entiendo yo. Y así lo ha entendido la Ilustre Municipalidad de Concepción. Esta institución cívica de mi ciudad natal, al designar a usted como digno e indiscutible depositario dei primer Premio de Literatura regional, ha cumplido con un alto deber, honrándose a sí misma, pues los señores regidores, más los miembros del Jurado discernidor, vieron claro y actuaron con talento y equidad, diría con honestidad, cosa que, desgraciadamente, no es pan de cada día ni atributo de los más.

Descubro ahora la razón del subconsciente - si razón cabe en él- que hizo vacilar mi primer intento de llegar hasta usted con mis congratulaciones. Eliminado el primer impulso, y razonado serenamente - corridos varios meses- quedo como al comienzo, cuando el veredicto del tribunal literario llegó hasta mí: ¡henchido de júbilo porque se hizo justicia! Y como el ser humano, tan complejo y contradictorio y tan lleno de vicios en el espíritu, carece de la aptitud suficiente para ser, en sus actos y en sus juicios, estrictamente justo, cuando actúa bajo los dictados de esta ansiada sabiduría de las sabidurías, entonces, a los que anhelamos y sufrimos por alcanzarla, nos nace una reconciliación con la humanidad y con el destino. Pues, adaptando un pensamiento de Montesquieu, diría que la justicia hecho a uno solo, es un bien hecho a todos.

Muere un año; nace otro. Muere una esperanza; nace otra. Que el nuevo año nazca en usted lleno de esperanzas para ser cuajadas en realidad en el desgranar de los días de 1954, para su propia felicidad y para felicidad de los que lo estiman.

Con todo afecto.-Ernesto Boero Lillo. 